\title{
Assessment of Light Microscopy, Rapid Diagnostic Test and Polymerase Chain Reaction on the Diagnosis of Malaria Infection in Infected Individuals in Southern Nigeria
}

\author{
T.O. Alade*, E.U. Eric and I. Arikekpar \\ Department of Medical laboratory Science, Niger Delta University, \\ Wilberforce Island, Bayelsa, Nigeria \\ *Corresponding author
}

\section{Keywords}

Light Microscopy, Rapid Diagnostic

Test and

Polymerase Chain

Reaction

Malaria

Nigeria

Article Info

Accepted:

10 January 2019

Available Online:

10 February 2019

\section{A B S T R A C T}

Rapid diagnosis and effective treatment of cases is the main objectives of control programmes in malaria endemic areas as malaria is one of the most deadly infections in some of the African countries. Its diagnosis is vital towards the effectiveness of antimalarial drug used. Routine microscopically examination, rapid diagnostic test and Polymerase chain reaction were compared. A total of Eight hundred and forty six positive samples were collected across the four Southern states (Bayelsa, Rivers, Edo and Delta) of Nigeria. Results: Out of the 192 samples screened from Bayelsa state 124(64.6\%) were positive for microscopy and $68(35.4 \%)$ negative, $57(29.7 \%)$ were positive for rapid diagnostic test (RDT) and 135(70.3\%) were negative, 150(78.1\%) were positive for PCR and $42(21.9 \%)$ negative. Out of the 218 samples screened from Rivers, 135(61.9\%) were positive for microscopy and $83(38.1 \%)$ negative, 90(41.3\%) were positive for RDT and 128 (58.7\%) negative, 150(68.8\%) were positive for PCR and 68(31.2\%) negative. Out of the 196 samples screened from Edo, 103(52.6\%) were positive for microscopy and 93(47.4\%) were negative, 63(32.1\%) were positive for RDT and $133(67.9 \%)$ were negative, 123(62.8\%) were positive for PCR and 73(37.2\%) negative. Out of the 240 samples screened from Delta, 121(50.4\%) were positive for microscopy and 119(49.6\%) were negative, $78(32.5 \%)$ were positive for RDT while $162(67.5 \%)$ were negative, $141(58.8 \%)$ were positive for PCR while $99(41.2 \%)$ were negative. PCR technique which is the most sensitive diagnostic tool though more expensive should be recommended also routine microscopy and RDT should not be withdrawn for prompt diagnosis and cost effectiveness.

\section{Introduction}

Malaria elimination has been a difficult task in the South-south endemic region. Series of strategies were being used to strengthen malaria control and to bring about malaria elimination. Such intervention is relied on specific and accurate diagnosis for effective treatment with antimalarial drugs especially for complicated cases of Plasmodium falciparum infections.

Microscopy and Rapid diagnostic tests were used in diagnosing malaria cases according to WHO recommendation which were universally accepted for prompt confirmation 
of the parasite (WHO, 2012). Presumptive treatment of malaria based on clinical diagnosis is relatively cheap but it is unreliable because of the existence of other non-malarial agent that caused overlapping symptoms which may lead to over-diagnosis. Wrong diagnosis may be detrimental to possible prescription and the patient may leave the hospital without right treatment (Reyburn et al., 2004).

Malaria poses different challenges in diagnosis across malaria endemic countries. New technology has been accepted by WHO standards that make comparisons of sensitivities and specificity among different methods. Accuracy of a clinical diagnosis could be presented by malaria season, age group and the endemicity. Rapid, sensitive and available diagnostic tool in identification of malaria parasite is significant in the management of malaria. Malaria spread, infection and death rate could be reduced drastically if rapid diagnosis and sufficient treatment is available (Manjurano et al., 2011). RDT provides rapid accuracy and prompt diagnosis to people at risk of malaria infection and also those that could not discover good laboratory for qualitative microscopic services (Manjurano et al., 2011).

In malaria-endemic regions, RDT usage is very important for the effective prescription and usage of antimalarial drugs based on the diagnosis apart from symptoms in these region, some of the antimalarial drugs distributed without diagnosis had been considered wasted due to lack of specific, prompt and accurate diagnosis (WHO, 2015).

The desire to use either microscopy or RDT is not far-fetched as the performance of both diagnostic tool depend on some conditions like transmission intensity, the prevalence of the infections, parasite density and parasite count in the blood (Hopkins, 2008). In an endemic area like ours, microscopy was reported to detect around $75 \%$ of malaria infections where in a low transmission area it can miss detection up to $80 \%$ (Okell et al., 2009) Clinical studies provided effective comparison between different test as well as the clarification on the feasibility and clinical relevance of using non microscopic method (Reyburn et al., 2004). However there is a noticeable reduction in Plasmodium falciparum transmission across Africa, malaria is still one of the public health problem by which children of less than age five were still more susceptible (Murray et al., 2012).

According to the discovery there is vital requirement to enhance the diagnostic tools in order to prepare for recovery of malaria infection. It is crucial to recognize the performance of different malaria diagnostic tests in different locations. There is a need to further discover the accurate diagnosis of malaria infection not only to lay emphasis on the signs and symptoms presented.

Microscopy is still the first diagnostic tool used for malaria diagnosis despite its low sensitivity and limited availability (Strom et al., 2013). Malaria microscopy is not as quick as RDT and it requires expertise to be able to ensure reliable results as the results may not be the same from different laboratories. RDT availability has made the diagnosis easy and prompt for identification of $\mathrm{P}$ falciparum infection in a region where laboratory facilities were limited and is generally recommended by WHO (Wilson, 2013). Microscopy and RDT were limited in their detection response in some circumstances where there are low parasite count (Mtove et al., 2011) Polymerase Chain reaction (PCR) is described as one of the most sensitive molecular tools that can identify parasites as minute as $0.01-0.2$ parasites/l of blood 
(Cordray et al., 2012). As there are different reports for microscopy and RDT, there are also different reports with PCR assays in respect to its sensitivity and (Lo et al., 2015).

Nevertheless PCR is highly sensitive, expensive and may not be used routinely in an area where resources in putting the technique in order may be limited. The evaluation of malaria diagnostic tests may vary depending on the malaria transmission geographical location. The study assessed the diagnostic features of the light microscopy, RDT and conventional PCR used to identify P. falciparum malaria infection among infected individual in some south-south states of Nigeria.

\section{Materials and Methods}

\section{Study site and subject}

The study was carried out at Molecular Laboratory of Niger Delta University, Wilberforce Island, Bayelsa between April and September 2015. The subjects 846 from Bayelsa, Rivers, Edo and Delta (Fig. 3). Ethical approval was sought for from the teaching hospitals. Sample Collection: Samples were collected into EDTA bottles, the blood was prepared for thick and thin slides for microscopy, for the RDTs assay and for PCR.

Sample Collection: Sample Collection: Samples were collected into EDTA bottles, the blood was prepared for thick and thin slides for microscopy, for the RDTs assay and for PCR.

\section{Thick and thin film for microscopy}

Both the films were prepared on slides but the thin film was fixed with methanol before they were stained with 5\% Giemsa solution for identification. A stained slide is regarded as negative if no parasite was seen in at least 100 fields of oil-immersion, high-power on the thick film, also the thick film was used for parasite density, the count was made against 200 white blood cells.

\section{Rapid diagnostic test}

SD Bioline malaria antigen Plasmodium falciparum Histidine rich protein 2 (HRP-2)based RDT, (Standard Diagnostics Limited, Korea) was used for detection of malaria infection. The test detects HRP-2 antigen from $P$. falciparum and P.vivax. The tests were performed and interpreted following manufacturer's instructions. PCR technique: DNA was extracted using the quick gDNA mini prep DNA extraction kit supplied by Inqaba Biotechnological, South Africa (12,000rpm) according to manufacturer's instruction and kept at -200c. The extracted DNA was amplified using nested PCR targeting as described by (Golassa et al., 2013).

\section{PCR technique}

DNA amplification was done by Thermal cycler 9700(Applied Biosystems (USA) The PCR products were resolved in $1.5 \%$ agarose gel stained with ethidium bromide alongside with100bp DNA ladder. The gels were visualized under UV transilluminator from BIO-RAD.

\section{Results and Discussion}

Findings from this study show that age group less than five years were more affected by malaria infection when compared with other individuals (Figure 1). Males were more infected than females (Figure 2). From each state, 192 samples were examined from Bayelsa, 124(64.6\%) were positive for microscopy, 57(29.7\%) were positive for RDT and 150(78.1\%) were positive for PCR, 
218 samples were examined from Rivers, $135(61.9 \%)$ were positive for microscopy, 90(41.3\%) were positive for RDT and 150(68.8\%) were positive for PCR, 196 samples were examined from Edo, $103(52.6 \%)$ were positive for microscopy, 63(32.1\%) for RDT and 123(62.8\%) were positive for PCR, 240 samples were examined from Delta, 121(50.4\%) were positive for microscopy, 78(32.5\%) were positive for RDT and 141(58.8\%) were positive for PCR. In overall, from the 846 samples from the states, $483(57.1 \%)$ were positive for microscopy, 288(34.0\%) were positive for RDT and 564(66.7\%) were positive for PCR (Table 1).

Table.1 Methods of identification of Malaria parasite

\begin{tabular}{|l|l|l|l|l|}
\hline States & Nos Examined & Microscopy & $\begin{array}{l}\text { Rapid } \\
\text { Diagnostic test }\end{array}$ & PCR \\
\hline Bayelsa & 192 & 124 & 57 & 150 \\
\hline Rivers & 218 & 135 & 90 & 150 \\
\hline Edo & 196 & 103 & 63 & 123 \\
\hline Delta & 240 & 121 & 78 & 141 \\
\hline Total & 846 & 483 & 288 & 564 \\
\hline
\end{tabular}

Figure.1 Distribution of malaria infection by age and by states

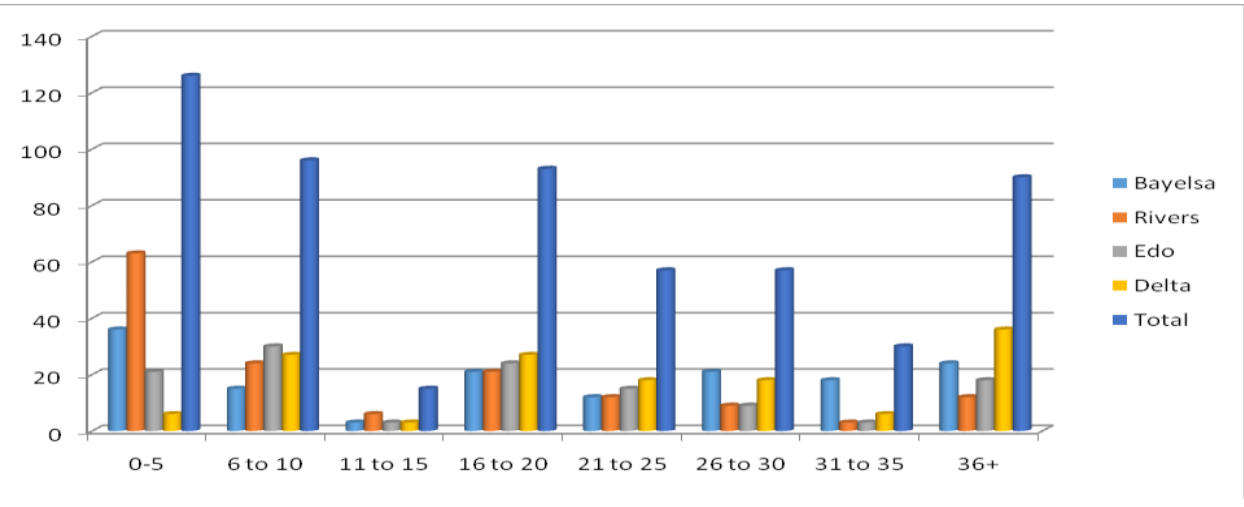

Figure.2 Distribution of malaria infected subjects by gender and by states

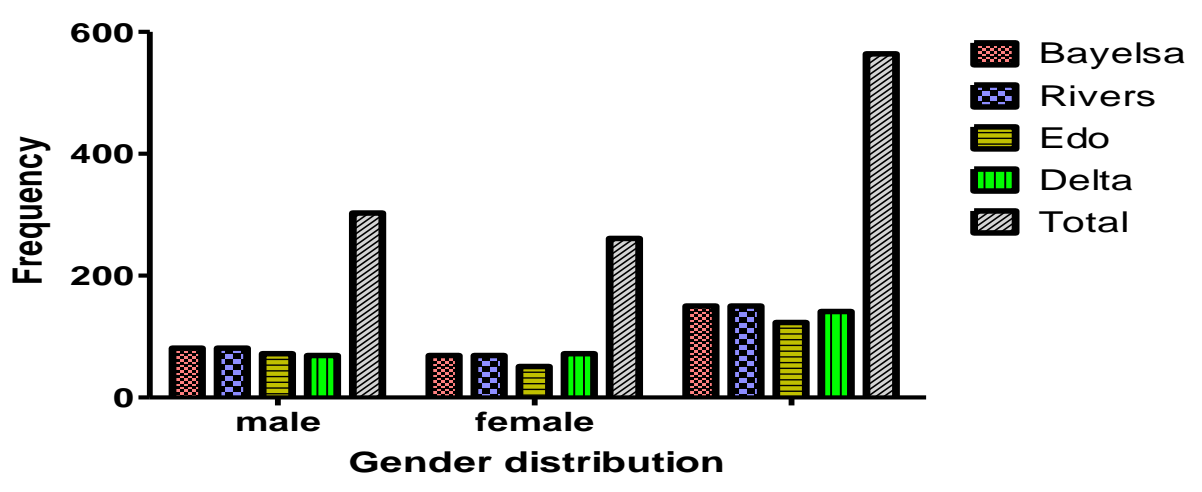


Figure.3 Study site

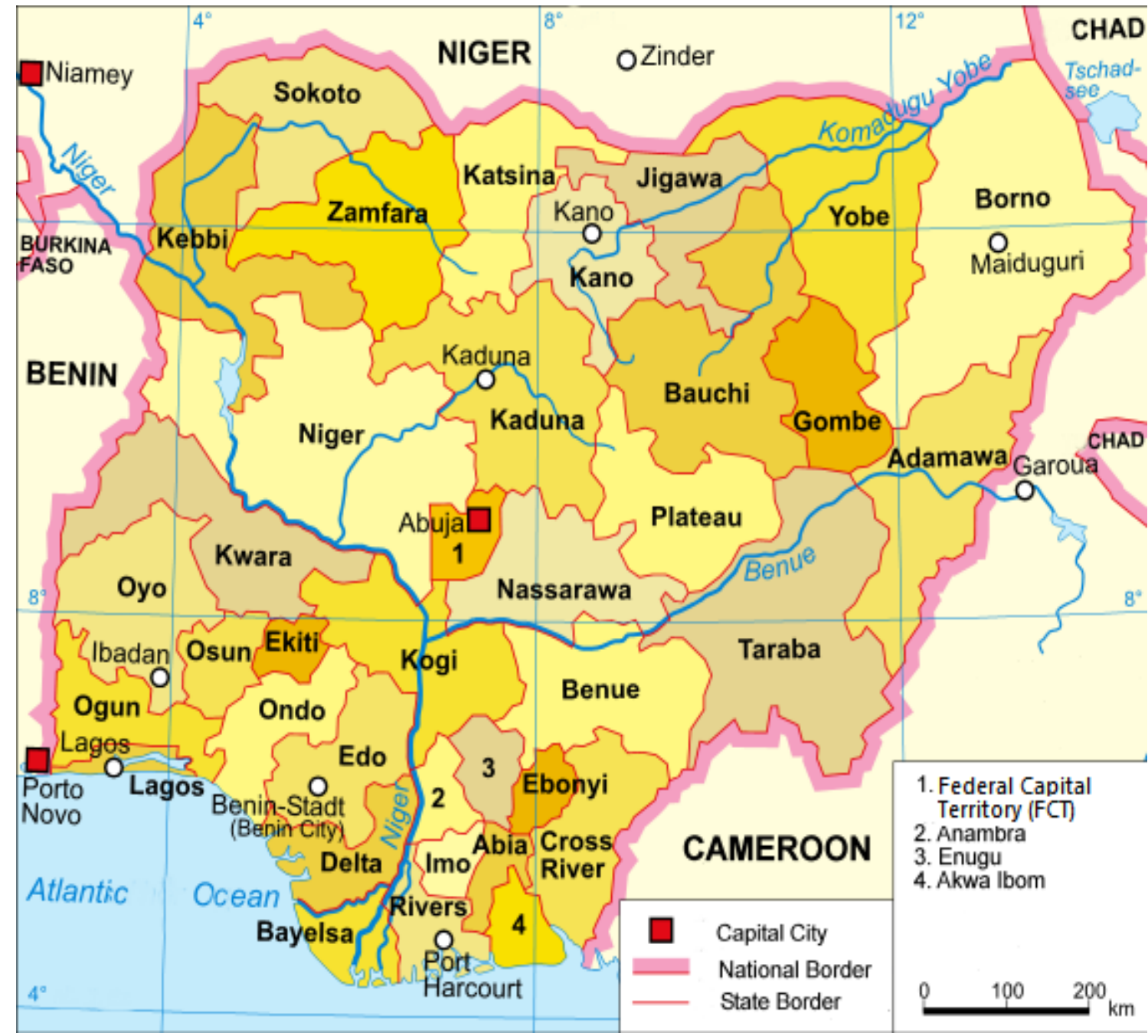

This study assessed the routine performance of the three main diagnostic tools to identify malaria parasite in malaria infection in some South-south states of Nigeria among children and adults attending the teaching hospitals. PCR performed better than microscopy and HRP-2-based malaria RDT. Overall, malaria prevalence was high with the different diagnostic tools used.

From the routine microscopy, the positive slides were confirmed to ascertain the positivity and the parasitaemia level. Microscopy is still found as the first diagnostic tool to be used for malaria parasite identification due to its simplicity and time saving characteristics. The diagnostic method were sensitive all-round the states ( $p>0.05)$.

This shows that the microscopy is still recognized as gold standard for method of identification of malaria parasite. Rapid diagnostic technique also was recommended to confirm further the positivity meanwhile in this study, the technique was least sensitive when compared with microscopy and PCR, the sensitivity value of malaria RDT was below the $95 \%$ threshold recommended by WHO even with these limitations RDTs is still recommended where access to 
microscopy is limited due to easy availability and its cost effectiveness (Harcut et al., 2013).

There are considerations to advance the malaria RDTs specificity and sensitivity for prompt and better controlling of malaria. In Bayelsa, RDT had the least sensitivity whereas it was most sensitive in Rivers. According to (Shakeley et al., 2013) the RDT has a good percentage for sensitivity more than PCR and microscopy which could not support findings from this study, the reason could be locational or geographical factor. Based on the PCR technique, some of the microscopy and RDT negative slides were positive with this diagnostic tool.

PCR and other molecular techniques were indicated to be the most sensitive diagnostic tool than microscopy and RDT (Mwigira et al., 2014). PCR efficacy and sensitivity regarding total eradication of malaria is still of concern due to cost and lack of technical man-power. Some of the limitations in these findings were the microscopy and RDT could not discover other species of Plasmodium apart from falciparum but nested PCR was able to differentiate among the species using species specific primers.

Plasmodium falciparum was the most prevalent while Plasmodium ovale and Plasmodium vivax also appeared as mixed infections with $P$. falciparum (Alade et al., 2018). The malaria infection prevalence was on a high side among individual in the states. Diagnosis of malaria still remains a challenge due to differences in the results from the three diagnostic methods.

In conclusion, microscopy and malaria RDT were not as sensitive as PCR therefore PCR technique is required in an endemic malaria region.

\section{References}

Alade T. Pughikumor D, Lawani E, Fabunmi B, and Tatfeng M (2018). Molecular detection of Plasmodium species in four Southern states of Nigeria. International Journal of Science and Research, 7(10): 296.

Bejon P, Andrews L, Hunt-Cooke A, Sanderson F, Gilbert S and Hill A (2006). Thick blood film examination for Plasmodium falciparum malaria has reduced sensitivity and underestimates parasite density. Malaria Journal.; 5:104.

Golassa L, Enweji N, Erko B, Aseffa A, Swedberg G (2013). Detection of a substantial number of sub-microscopic Plasmodium falciparum infections by polymerase chain reaction: a potential threat to malaria control and diagnosis in Ethiopia. Malaria Journal; 12: 352

Harchut K, Standley C, Dobson A, Klaassen B, Rambaud-Althaus C and Althaus F (2013). Over-diagnosis of malaria by microscopy in the Kilombero Valley, Southern Tanzania: an evaluation of the utility and cost-effectiveness of rapid diagnostic tests. Malaria Journal. Pp. 12: 159.

Hopkins, H., Bebell, L., Kambale, W., Dokomajilar, C., Rosenthal, P. and Dorsey, G. (2008). Rapid diagnostic tests for malaria at sites of varying transmission intensity in Uganda. Journal of Infectious Disease, 197. 510518.

Lo E, Zhou G, Oo W, Afrane Y, Githeko A and Yan G (2015): Low parasitemia in submicroscopic infections significantly impacts malaria diagnostic sensitivity in the highlands of Western Kenya. PLoS ONE., 10:e0121763.

Manjurano A, Okell L, Lukindo T, Reyburn H, Olomi R and Roper C, (2011). Association of sub-microscopic malaria 
parasite carriage with transmission intensity in north-eastern Tanzania. Malaria Journal; 10:370.

Mtove G, Nadjm B, Amos B, Hendriksen I, Muro F and Reyburn H (2011). Use of an HRP2-based rapid diagnostic test to guide treatment of children admitted to hospital in a malaria-endemic area of north-east Tanzania. Tropical Medicine International Health.16:545-50.

Murray C, Rosenfeld L, Lim S, Andrews K, Foreman K and Haring D, (2012) Global malaria mortality between 1980 and 2010: a systematic analysis. Lancet.; 379: 413-31.

Mwingira F, Genton B, Kabanywanyi A, Felger I (2014). Comparison of detection methods to estimate asexual Plasmodium falciparum parasite prevalence and gametocyte carriage in a community survey in Tanzania. Malaria Journal; 13:433.

Okell L, Bousema T, Griffin J, Ouedraogo A and Ghani A and Drakeley C (2015): Factors determining the occurrence of submicroscopic malaria infections and their relevance for WHO: World malaria report. Geneva: World Health Organization; 2015.
Reyburn H, Mbatia R, Drakeley C, Carneiro I, Mwakasungula E, Mwerinde O, Saganda K, Shao J, Kitua A, Olomi R, Greenwood B, Whitty C. (2004): Over diagnosis of malaria in patients with severe febrile illness in Tanzania: a prospective study. Biomedical Journal. 329: 1212-1217.

Shakely D, Elfving K, Aydin-Schmidt B, Msellem MI, Morris U and Omar R, (2013): The usefulness of rapid diagnostic tests in the new context of low malaria transmission in Zanzibar. PLOS ONE. 8:72912.

Strom G, Haanshuus C, Fataki M, Langeland $\mathrm{N}$ and Blomberg B (2013). Challenges in diagnosing paediatric malaria in Dares Salaam, Tanzania. Malaria Journal; 12: 22.

WHO (2012). Universal access to malaria diagnostic testing. Geneva: World Health Organization;

WHO (2015): World malaria report. Geneva: World Health Organization;

Wilson M (2013). Laboratory diagnosis of malaria: conventional and rapid diagnostic methods. Archea Pathology Laboratory Medicine.; 137: 805-811.

\section{How to cite this article:}

Alade, T.O., E.U. Eric and Arikekpar, I. 2019. Assessment of Light Microscopy, Rapid Diagnostic Test and Polymerase Chain Reaction on the Diagnosis of Malaria Infection in Infected Individuals in Southern Nigeria. Int.J.Curr.Microbiol.App.Sci. 8(03): 1037-1043. doi: https://doi.org/10.20546/ijcmas.2019.803.126 